\title{
Low Peak to Average Power Ratio Cyclic Golay Sequences for OFDM Systems
}

\author{
Masoud Olfat and K. J. Ray Liu \\ Electrical and Computer Eng. Department, University of Maryland, College Park, MD 20742
}

\begin{abstract}
In this paper, the cyclic shifts of low Peak to Average Power Ratio (PAPR) codes used in OFDM transmission is proposed. They comprise a superclass of Golay Complementary Codes (GCC) with the same level of PAPR (viewed by the discrete OFDM symbols). These codes achieve higher information rate with the expense of lower error correction capabilities. We have proposed a framework for constructing these codes out of GCC. The framework can be applied to obtain the cyclic shift of any code represented by Boolean algebraic functions. An efficient transmission scheme is proposed such that the coding scheme can be switched between the Golay and cyclic Golay codes, based on a feedback from the receiver to perform a better tradeoff between the coding rate, and Bit Error Rate (BER).
\end{abstract}

\section{INTRODUCTION}

Supporting high data rate and acceptable quality transmission in wireless media, requires high SINR at the receiver side. However, the power limitation in mobile devices has made this a challenging goal. As a result, efficient utilization of the power in mobile devices, is an attractive area of research. OFDM is a parallel data transmission scheme that has recently attracted lots of interest. One of the problems of OFDM is its high Peak to Average Power Ratio (PAPR) [1]. Usually, the transmitters are constrained to a limited peak power. This in turn reduces the average power allowed under OFDM. In addition, to prevent the inter-channel interference among subcarriers, the transmitter amplifier must operate in a linear region. A large PAPR may force the amplifier to operate in nonlinear region. This may reduce the mobile battery lifetime.

The conventional solution to this problem is to use a linear amplifier or to back-off the operating point of nonlinear amplifier, both result in efficiency penalty. Another solution [2] is to simply clip the signal before amplification. This approach causes performance degradation.

One of the recent and efficient approaches in this area uses Golay Complementary Sequences (GCS) [3], resulting in OFDM signals with PAPR at most 2. The correlation properties of these codewords have made them a suitable choice for several applications like CDMA systems. The IEEE802.11 standard committee has adopted Complementary Code Keying (CCK) signals, that are basically QPSK GCS, as the physical layer of Wireless LAN IEEE802.11b standard. Davis and Jedwab in their landmark paper [4] obtained a large set of length $2^{m}$ binary Golay Complementary Pairs (GCP) from certain second order cosets of the first order Reed-Muller [5] codes. By allowing higher
PAPR, they were able to guarantee higher coding rates. Paterson [6] generalized Davis and Jedwab's results and used $q$-ary instead of $2^{h}$-ary alphabets (with $q$ even). The authors in [7] found a trade-off between the PAPR, the data rate and the minimum distance of the codebook. In other words, given the data rate and minimum distance, they found a lower bound for the PAPR that increases with increasing data rate. As a matter of fact, one of the major drawbacks of exploiting block codes (including Golay codes) in OFDM, despite their low PAPR, is their low data rate.

In this paper we will set an upper bound for the PAPR $(3 d B)$ and provide some trade-offs between the coding rate and the error correction capabilities of the OFDM code, by developing the concept of cyclic Golay codes. We will present a method for constructing these codes out of Golay codes. This construction method is suitable for generating the cyclic shift of any code described by means of Boolean functions. We will show that if cyclic Golay codes are applied to the IFFT block in an OFDM system, the PAPR defined over discrete symbols is bounded up by $3 d B$. We will also present a trade-off between the coding rate and minimum distance of the code. The error correction capabilities of these codes are in general lower than GCC. However, if the signal to noise ratio in an environment is above a threshold, we might be able to tolerate lower distance codes for higher rates. We will present an OFDM transmission system that demonstrates the trade-off between the bit error rate and the coding rate. It is worthwhile to mention that although this paper does not offer a significant rate increase over the GCC, it outlines a methodology to generate the cyclic shift of any code, presented by Boolean algebraic functions. We have provided efficient decoding scheme for these codes in [8].

The remainder of this paper is organized as follows: Section II outlines the definition of cyclic Golay codes and their properties. In Sections III and IV, we'll find construction method for binary and non-binary cyclic Golay codes out of GCC. In Section V, we will demonstrate an OFDM transmission system using these codes and present some simulation results and finally, Section VI concludes the paper. II. CyClic Golay SEQuences

The instantaneous envelope power of an $N$-tones OFDM signal is

$$
p_{x}(t)=\sum_{i=0}^{N-1} \sum_{u=0}^{N-1} x[i] x^{*}[u] \exp [j 2 \pi(i-u) \Delta f t],
$$

where $x$ is an $N$-valued complex sequence applied to IFFT block, $a^{*}$ denotes the complex conjugate of the complex 
number $a, j=\sqrt{-1}$, and $\Delta f$ is the frequency separation between OFDM subcarriers. The actual peak of OFDM time domain signal depends on the pulse shaping and the low-pass filter we use after the IFFT block. It is obvious that this peak is in general different from the peak of the samples of the OFDM signal at the multiples of $\frac{1}{N \Delta f}$. However, there is a direct relation between the peak of the continuous OFDM signal and the maximum of the discrete OFDM symbols sampled at the multiples of $\frac{1}{N \Delta f}$ [9]. By some proper time shaping, we can consider the PAPR of the discrete sequence obtained after IFFT operation, as a measure of the PAPR of the OFDM signal. In the sequel, we define the PAPR of a codeword $x$ to be

$$
P A P R(x)=\frac{1}{P_{x}} \max _{k}\left[\sum_{i=0}^{N-1} x[i] x^{*}[u] e^{\frac{j 2 \pi(i-u) k}{N}}\right],
$$

where $P_{x}=\|x\|^{2}$ is the energy of the codeword $x$. By defining the auto-correlation of an $N$-valued complex sequence $x$ with replacement $l$ to be $A_{l}(x)=\sum_{i=0}^{N-l-1} x[i+$ $l] x^{*}[i]$, the power of the $k^{\text {th }}$ OFDM channel symbol can be restated as:

$$
p_{x}[k]=A_{0}(x)+\sum_{j 2 \pi k}^{N-1} A_{l}(x) w_{k}^{l},
$$

where $w_{k} \triangleq e^{\frac{j 2 \pi k}{N}}$ is the $N^{t h l \mid}$ root of unity. Note that $A_{0}(x)$ is actually the same as $P_{x}$, the power of the code $x$, and by Parseval equation, this is the same as the average power of OFDM channel symbols.

Definition 2.1: Two $N$-valued complex sequences $x$ and $y$ are called Golay Complementary Pairs $(G C P)$ if $A_{l}(x)+$ $A_{l}(y)=0, \quad \forall l \neq 0$. Each of the sequences $x$ and $y$ is called Golay complementary sequence.

Since the power of each channel symbol is non-negative, if the sequences $x$ and $y$ are GCP and have the same power $\left(A_{0}(x)=A_{0}(y)\right)$, it is straightforward to see that $P A P R(x) \leq 2=3 d B$ and $P A P R(y) \leq 3 d B$. Consequently, if we choose the codewords from a set of Golay sequences, the PAPR is bounded up by $3 \mathrm{~dB}$.

For simplicity, we will denote $" k \bmod n "$ by $" k \% n "$ in the following definition and what comes hereafter.

Definition 2.2: The cyclic auto-correlation of an $N$ valued complex sequence $x$, with nonzero replacement $l$, is defined as $C A_{l}(x)=\sum_{i=0}^{N-1} x^{*}[i] x[(i+l) \% N]$.

Using this definition, one can see that

$$
C A_{l}(x)=A_{l}(x)+A_{N-l}^{*}(x) .
$$

Definition 2.3: Two $N$-valued complex sequences $x$ and $y$ are called cyclic Golay complementary pairs if $C A_{l}(x)+$ $C A_{l}(y)=0, \quad \forall l \neq 0$. Each of the sequences $x$ and $y$ is called Cyclic Golay Complementary Sequence (CGCS).

Using (3), it is obvious that if two sequences are Golay pairs, they are cyclic Golay pairs, too. The following theorem is easily followed from (2) and (3):

Theorem 2.1: The PAPR (viewed by discrete symbols) of cyclic Golay sequences is upper bounded by $3 \mathrm{~dB}$.

Theorem 2.1 states that the number of codewords achieving a PAPR at most equal to $3 \mathrm{~dB}$ (in discrete domain), is more than just the number of Golay sequences. This is translated to higher coding rates.

If $x$ is an $N$-sized sequence, we denote its cyclic $l$-shift by $x^{l}(0 \leq l \leq N-1)$. The $k^{t h}$ element of $x^{l}$ is given by $x^{l}[k]=x[(k+l) \% N]$.

Lemma 2.1: The property of being cyclic Golay is preserved under any cyclic $l$-shift of a sequence with size $\mathrm{N}$. Thus, if two sequences are cyclic Golay sequences, their shifted version by any replacement $l$ are cyclic Golay, too.

Theorem 2.1 can be generalized for non-Golay sequences in the following way:

Theorem 2.2: The PAPR achieved by any cyclic shifted versions of a sequence $x$ is the same as the PAPR achieved by the sequence $x$ itself.

In [10], we have listed several transformations that when performed on Golay pairs, the resultant pairs are still GCP. These transformation are reflection with respect to the origin, with respect to both axes, with respect to the bisectors of all regions, and the rotation of one or both sequences. The same was true for the concatenation and interleaving of Golay pairs, reversing each sequence in Golay pairs, and alternatively multiplying the elements of each sequence by -1 . Considering equation (3), we can deduce that the property of being cyclic Golay pairs is invariant under each of these transformations.

\section{CONSTRUCTION OF BINARY CYCLIC GOLAY CODES}

Let's correspond a generalized Boolean function of $m$ variables, $f\left(x_{1}, x_{2}, \ldots, x_{m}\right): \mathcal{Z}_{2}^{m} \longrightarrow \mathcal{Z}_{2^{h}}$, to a sequence f of length $2^{m}$, by listing the values taken by the function $f$ as $\left(x_{1}, x_{2}, \ldots, x_{m}\right)$ ranges over all its $2^{m}$ combinations in lexicographic order. In other words, if $\left(i_{1}, i_{2}, \ldots, i_{m}\right)$ is the binary representation of integer $i$, i.e. $i=\sum_{k=1}^{m} i_{k} 2^{k-1}$, the $i^{t h}$ element of the sequence $\mathbf{f}$ is $f\left(i_{1}, i_{2}, \ldots, i_{m}\right)$.

We assume that the elements of the codeword to be transmitted using an OFDM system, are chosen from an equal-energy constellation like QPSK or 8-PSK. Therefore, without loss of generality, the elements of a codeword $y$ can be written as $y[i]=\exp \left(\frac{j 2 \pi}{2^{h}} a_{i}\right)$, where $a_{i}$ is chosen from a $2^{h}$-ary alphabet, $\mathcal{Z}_{2^{h}}$. As a result, constructing the sequence $a$, will provide the sequence $y$. Using these notations, the main result of [4] states that if

$$
f=\left[2^{h-1} \sum_{k=1}^{m-1} x_{\pi(k)} x_{\pi(k+1)}+\sum_{k=1}^{m} c_{k} x_{k}+c\right],
$$

where additions are in modula $2^{h}, \pi$ is a permutation of the symbols $\{1,2, \ldots, m\}$, and $c, c_{k} \in \mathcal{Z}_{2^{h}}$, the sequences $f$ and $\left(f+2^{h-1} x_{\pi(1)}+c^{\prime} \bmod 2^{h}\right)$ comprise a Golay pair of length $2^{m}$ over $\mathcal{Z}_{2^{h}}$, for any $c^{\prime} \in \mathcal{Z}_{2^{h}}$.

For $h \geq 1$ and $0 \leq r \leq m$, the $r^{t h}$ order linear ReedMuller code of length $2^{m}$ over $\mathcal{Z}_{2^{h}}, R M_{2^{h}}(r, m)$, is defined to be generated by all monomials in the $x_{i}$ of degree at most $r$. Using this definition, the authors in [4] have restated their main result as: Each of the $\frac{m !}{2}$ cosets of $R M_{2^{h}}(1, m)$ having a coset representation of the form $2^{h-1} \sum_{k=1}^{m-1} x_{\pi(k)} x_{\pi(k+1)}$ comprises $2^{h(m+1)}$ Golay 


\begin{tabular}{|c||c|c|c|}
\hline Shifts/Coset Rep. & $x_{1} x_{2}+x_{2} x_{3}$ & $x_{1} x_{3}+x_{2} x_{3}$ & $x_{1} x_{3}+x_{1} x_{2}$ \\
\hline \hline$l=1$ & \multicolumn{2}{|c|}{$c_{3}=0 \&\left(c_{2}=1\right.$ or $\left.c_{2}=3\right)$} & $c_{3}=0 \&\left(c_{2}=0\right.$ or $\left.c_{2}=2\right)$ \\
& $c_{3}=2 \&\left(c_{2}=0\right.$ or $\left.c_{2}=2\right)$ & $c_{3}=2 \&\left(c_{2}=1\right.$ or $\left.c_{2}=3\right)$ \\
\hline$l=2$ & $c_{3} \% 2=0$ & $c_{3} \% 2=1$ \\
\hline$l=3$ & $c_{3}=0 \&\left(c_{2}=0\right.$ or $\left.c_{2}=2\right)$ & $c_{3}=0 \&\left(c_{2}=1\right.$ or $\left.c_{2}=3\right)$ \\
$c_{3}=2 \&\left(c_{2}=0\right.$ or $\left.c_{2}=2\right)$
\end{tabular}

LIST OF REPEATED GOLAY SEQUENCES UNDER CYCLIC SHIFTS FOR $m=3$ AND $h=2$

sequences over $\mathcal{Z}_{2^{h}}$ of length $2^{m}$, where $\pi$ is a permutation of the symbols $\{1,2, \ldots, m\}$.

By varying $c_{k}$ 's and $c$ over $\mathcal{Z}_{2^{h}}$ in (4), this theorem generates $\frac{m !}{2} 2^{h(m+1)}$ Golay sequences of length $2^{m}$. So, the coding rate of Davis-Jedwab construction is $\frac{h(m+1)+\log _{2}\left(\frac{m !}{2}\right)}{h 2^{m}}$. If we start from one of the their Golay sequences with size $2^{m}$ over $\mathcal{Z}_{2^{h}}$, and make a cyclic shift, the resultant sequence is cyclic Golay. This construction can create $2^{m}$ cyclic Golay sequences out of each Golay sequence by $l$-shifting the original sequence with $0 \leq l \leq 2^{m}-1$. However, some of these newly generated sequences are also part of original Golay sequences that can be created by different values of $c_{k}$ 's and $c$ in (4). As a result, we need to carefully develop a structure for constructing the cyclic Golay sequences. In what follows, we will design a framework for obtaining the cyclic shifts of a sequence, presented by Boolean functions. To this end, we start from the field $\mathcal{Z}_{2}$ with the addition defined modula 2 . We define the basis codeword $\left\{x_{n} \mid n=1 \ldots m\right\}$ to have 1 in positions $k$ where $k \% 2^{n} \geq 2^{n-1}$ and zero otherwise. By considering the relation $(t \% a b) \% b=t \% b$, we can represent the cyclic $l$-shift of $x_{n}$ as:

$$
x_{n}^{l}[k]= \begin{cases}0 & \text { if }(k+l) \% 2^{n}<2^{n-1}, \\ 1 & \text { if }(k+l) \% 2^{n} \geq 2^{n-1} .\end{cases}
$$

In the sequel, all additions are performed modula 2 and all codeword products are Hadamard product, unless otherwise stated. After some mathematical manipulations, the following Lemma is proved:

Lemma 3.1: The cyclic $2^{k}$-shift of a basis codeword $x_{n}$, when $n>1$ and $0 \leq k<n-1$, is

$$
x_{n}^{2^{k}}=x_{n}+\prod_{i=k+1}^{n-1} x_{i} \text {. }
$$

Moreover, $x_{n}^{2^{n-1}}=1+x_{n}$, and $x_{n}^{2^{k}}=x_{n}$ for $k \geq n \geq 0$ and all $n$.

Lemma 3.2: The following relations hold:

- For $2^{n-1} \leq l \% 2^{n}$, the $l$-shift of $x_{n}$ is $x_{n}^{l}=1+$ $x_{n}^{l \% 2^{n-1}}$.

- For $l \geq 2^{n}$, the $l$-shift of $x_{n}$ is $x_{n}^{l}=x_{n}^{l \% 2^{n}}$.

- If $l=2^{a}+2^{b}$ with $n-1>a>b$ we have

$$
x_{n}^{l}=x_{n}+\left(x_{a+1}+\prod_{i=b+1}^{a+1} x_{i}+\prod_{i=b+1}^{a} x_{i}\right) y_{n},
$$

where $y_{n}$ is 1 for $a=n-2$ and $\prod_{i=a+2}^{n-1} x_{i}$ for $a<n-2$.
Also, a Boolean function can be found for $x_{n}^{l}$ with $l=$ $2^{a}+b$, by replacing each $x_{n^{\prime}}$ in $x_{n}^{b}$, by $x_{n^{\prime}}+\prod_{i=a+1}^{n^{\prime}-1} x_{i}$.

The following lemma, states the counterpart of Lemma 3.1 for right cyclic shifts.

Lemma 3.3: For $n \geq 1$ and $0 \leq k<n-1$, the right cyclic $2^{k}$-shift of a basis codeword $x_{n}$, is

$$
x_{n}^{-2^{k}}=x_{n}+\prod_{i=k+1}^{n-1}\left(1+x_{i}\right) .
$$

Also, $x_{n}^{-2^{n-1}}=1+x_{n}$ and $x_{n}^{-2^{k}}=x_{n}$ for $k \geq n \geq 0$. Finally, the following Lemma relates the left cyclic $\left(2^{n-1}\right.$ l)-shift of $x_{n}$ to the right cyclic l-shift of the basis codewords.

Lemma 3.4: For $1 \leq l<2^{n-1}$, the left cyclic $\left(2^{n-1}-\right.$ l)-shift of $x_{n}$, is

$$
x_{n}^{2^{n-1}-l}=1+x_{n}^{-l} .
$$

\section{Cyclic Shift OF NON-Binary CODES}

If the Boolean functions are defined over $\mathcal{Z}_{2^{h}}$, there is an ambiguity over modula- 2 addition and modula- $2^{h}$ additions. This ambiguity can be avoided by using the relation $c[(x+y) \% 2]=(c x+c y-2 c x y) \% 2^{h}$, where $c$ is a constant number defined over $\mathcal{Z}_{2^{h}}$ and $x$ and $y$ are two binary codewords. By induction, we can generalize this relation for $m$ sequences $f_{i}(i=1, \ldots m)$.

As an application of this framework, we will construct the cyclic Golay code of size 8 over $\mathcal{Z}_{4}$. The Golay coset representations of $R M_{4}(1,3)$ in $R M_{4}(2,3)$, shown in (4), are $2\left(x_{1} x_{2}+x_{2} x_{3}\right), 2\left(x_{1} x_{3}+x_{2} x_{3}\right)$, and $2\left(x_{1} x_{2}+x_{1} x_{3}\right)$. Table I shows the Golay sequences that are repeated under cyclic shifts. The sequences are categorized based on the coset representative and the coefficient values from $\mathcal{Z}_{4}$. These values are obtained by applying the above mentioned framework to each basis codeword. As an example, when the coset representative is $2\left(x_{1} x_{2}+x_{2} x_{3}\right)$, the requirements for the $3^{\text {rd }}$ cyclic shift of the Golay sequence represented in (4) to be Golay (and therefore the shift does not create a new codeword) are

- $\left(2 c_{3}=0 \bmod 4\right)$ to delete the term $x_{1} x_{2} x_{3}$,

- One of the three terms " $-c_{3}-2 c_{2}$ " or " $2-2 c_{3} "$ or " $2-2 c_{3}$ " must be equal to zero, to create a valid second order coset.

- The other two nonzero terms must be equal to 2 . 


\begin{tabular}{|c||c|c|c|}
\hline Coeff. / Coset & $x_{1} x_{2}+x_{2} x_{3}$ & $x_{1} x_{3}+x_{2} x_{3}$ & $x_{1} x_{3}+x_{1} x_{2}$ \\
\hline \hline$c_{3}=0 ; c_{2} \% 2=0$ & None & $l=1,2,3$ & $l=2,3$ \\
\hline$c_{3}=0 ; c_{2} \% 2=1$ & None & $l=2$ & $l=1,2$ \\
\hline \hline$c_{3}=1$ & $l=1,2,3,5,6,7$ & None & $l=1,3,5,7$ \\
\hline \hline$c_{3}=2 ; c_{2} \% 2=0$ & None & $l=2$ & None \\
\hline$c_{3}=2 ; c_{2} \% 2=1$ & None & $l=1,2,3$ & None \\
\hline \hline$c_{3}=3$ & \multicolumn{4}{|c}{ None } \\
\hline \multicolumn{4}{|c}{ TABLE II }
\end{tabular}

LIST OF NON-REPEATED CYCLIC SHIFTS ON GOLAY SEQUENCES.

These requirements are translated into $c_{3} \% 2=0$. Moreover, if $c_{3}=0$ then $c_{2} \% 2=0$, and if $c_{3}=2$ then $c_{2} \% 2=1$. Therefore, out of 256 Golay sequences generated by this coset, the $3^{\text {rd }}$ cyclic shift of only 64 sequences are repetitions of Golay codes. The last row of Table I, shows the total number of cyclic Golay sequences created by each cost representative that are not Golay. Considering all 3 cosets, the cyclic shifts generate 3072 new sequences. However, some of the newly generated sequences by a coset representative can be created by some other cosets with different values of $c_{k}$ 's. For example, when $c_{3}=3$, all of the sequences generated by cyclic shifts of Golay codewords are similar to the ones created by $c_{3}=1$, with different values of $c_{1}, c_{2}$, and $c$, and different coset representatives. To find this out, we need to create a table containing all of the coefficients for each coset representatives, and each shift, for different values of $c_{3}$. By deleting the similar columns, we can find the non-repeated cyclic Golay sequences. We have performed such inspection for $m=3$ and $h=2$. The result shows that 1024 of these cyclic Golay sequences are non-repeated and can be generated from Golay sequences, using the procedure presented in Table II.

The rate of size 8 Golay sequences over $\mathcal{Z}_{4}$ is $\frac{\log _{2}\left(\frac{3 !}{2} 2^{2(3+1)}\right)}{2 \times 2^{3}}=0.599$, while the rate of size 8 cyclic Golay sequences over $\mathcal{Z}_{4}$ is $\frac{\log _{2}\left(1024+\frac{3 !}{2} 2^{2(3+1)}\right)}{2 \times 2^{3}}=0.690$.

The cyclic Golay code we generated, is clearly nonlinear. Therefore, we cannot define a minimal generator polynomial for generating this code. It is known that a linear cyclic code created by polynomials cannot be of size $2^{m}$ and our code is of size $2^{m}$. Massey [11] has introduced cyclic Reed-Muller codes by puncturing the first column of the generator matrix and reordering the first order rows to create $m$-sequences. The size of the codewords in this case is $2^{m}-1$.

The major drawback of cyclic Golay code is the low Hamming and Lee distances of the code. It is proved in [5] and [4] that the Hamming distance and Lee distance of the $R M_{2^{h}}(r, m)$ are both $2^{m-r}$. Therefore, the Hamming and Lee distance of the code defined in (4) are both $2^{m-2}$. The cyclic Golay code defined in this section, is in general a subset of $R M_{2^{h}}(m, m)$, and therefor, in general has a very low distance. However, by reducing the coding rate, we can increase the distances, while maintaining the same upper bound for the PAPR. This trade-off can be explained by an example. Take $m=4$, and $h=2$. If we start from (4) with the coset representation $x_{1} x_{2}+x_{2} x_{3}+x_{3} x_{4}$, and find the Boolean function representation of the cyclic 1-shift of each codeword, by avoiding $c_{4} \% 2=1$, the resulting code is in $R M_{4}(3,4)$, instead of $R M_{4}(4,4)$.

\section{SySTEM CONFIGURATION AND SIMULATION}

For the comparison of bit error rate, we used an OFDM system with $m=3$ and 4 , corresponding to 8 and 16 subchannels. A white Gaussian noise with variance 0.5 per dimension was assumed at each subchannel. The symbols were chosen from $\mathcal{Z}_{4}$, corresponding to $h=2$. The bit error rate versus SNR for Golay and cyclic Golay schemes is shown in Fig. 1.a for AWGN channels. It is obvious that the cyclic Golay codes with $m=4$, order 4 and $m=$ 3 , order 3 have the worst bit error rates, and the Golay code with $m=4$ has the best performance. The second order cyclic Golay code with $m=4$ performs better than other codes. As the difference between the dimension of the code and the order is decreased, the bit error rate of the code increases. This is attributed to the fact that the cyclic Golay code is a subset of $R M_{2^{h}}(r, m)$. Note that, the difference between the BER's are better observed at high SNRs. Fig. 1.b shows the rate we obtained for these codes. The following facts can be seen from this figure. First, the code with $m=4$, normally have lower rate than the cases with $m=3$. Second, for each dimension, the pure Golay code has the worst coding rate. Third, as the difference between the dimension and the order of cyclic Golay code decreases, the code can achieve better coding rate. Finally, although the cyclic Golay code can result in different rates at different OFDM block, the rate of the code in a long frame size is almost constant for all values of SNR. These two figures show the trade-off between the coding rate and the coding distance, while the PAPR is bounded up to $3 d B$.

The results for the 2-path fading channels is shown in Fig. 2.a. Both paths have a Raleigh envelope and the delay equal to the OFDM symbol duration divided by the number of subchannels. We have also considered the same white Gaussian noise as in Fig. 1 in these figures. In this figure we assume that the receiver knows the channel characteristics. We can observe the same trend as in Fig. 1.a, of course with lower bit error rates. We saw that the cyclic Golay code can perform higher coding rate at the expense of lower distance. However, in high SINR environment, we might be able to tolerate lower distance codes. We can use an OFDM system, with adaptive coding scheme. If the SINR is above a threshold, we use cyclic Golay codes at the transmitter to transmit higher data rates. If the SINR is below the threshold, we switch to the Golay codes having better error correcting property, but lower rates. The threshold can be chosen according to the upper bound of BER we can tolerate. We use this system along with $m=4$, for our simulation purposes in Figs. 3.a and 3.b. We assume that the SNR of the received signal changes randomly according to a uniform distribution. Notice that, the $x$-axis in these figures shows the SNR threshold on which we switch the coding scheme, not the actual SNR. The actual SNR is changed from one OFDM block to 

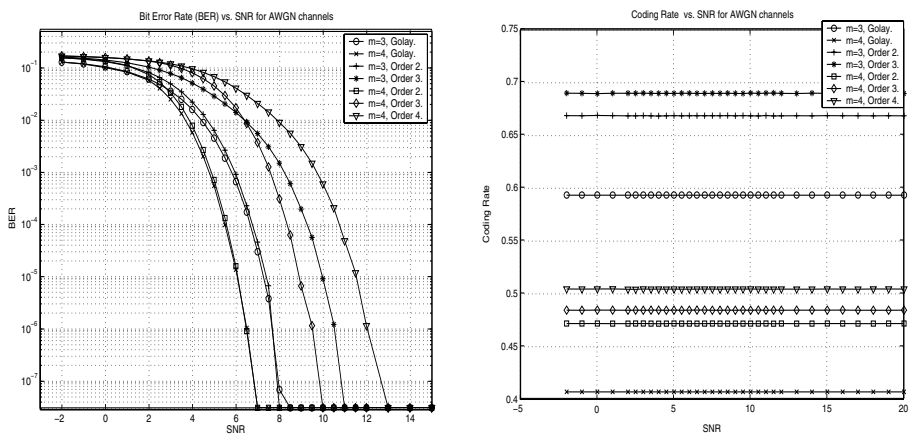

Fig. 1. (a) BER vs. SNR (b) Coding rate vs. SNR, for AWGN channels
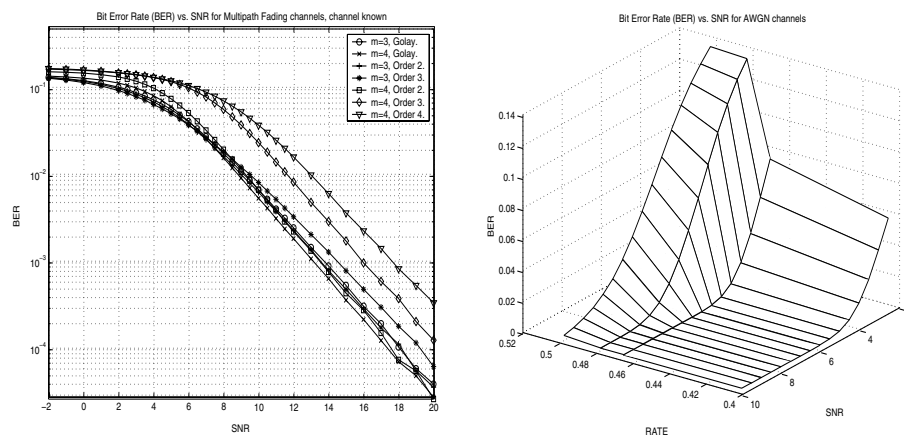

Fig. 2. BER vs. SNR for fading channels (b) BER vs. rate for fixed values of SNR for AWGN channels, when $m=4$ channel

another, according to a uniform distribution. When the threshold is set to the lowest value, the Golay codes are never used, and therefore we expect poor BER performance with a better coding rate. As the threshold becomes larger, the cyclic Golay code is less used and therefore the BER tends to be lower. When the SNR is set at the highest value, we use the Golay code, all the time.

Fig. 2.b shows the changes in bit error rate versus the coding rates for $m=4$, when the SNR is kept constant. The figure is in agreement with our expectation that for a fixed SNR, as the rate increases the code performs higher bit error rate. For example, by fixing the SNR at $6 d B$, as the coding rate goes from 0.40625 to 0.50358 , the bit error rate is increased from $5.4 \times 10^{-4}$ to $6.7 \times 10^{-2}$.

\section{CONCLUSION}

We have introduced the concept of cyclic Golay codes and shown that, with appropriate time shaping, they maintain the same level of PAPR as the Golay codes. Moreover, we have shown that the set of cyclic Golay codes is a superset of Golay codes and therefore results in higher coding rate. The increase in the coding rate is about $22 \%$ for a system with 16 tones. We have designed a construction method to find the cyclic shift of any code represented by Boolean algebraic forms. The cyclic shifts of the Golay second order cosets of the first order Reed-Muller codes generated by our construction has a low Hamming and Lee distance. However, we have introduced a trade-off between the coding rate and the distance of the code. An OFDM system, with a SNR threshold is introduced that according to the SNR of the received signal switches between Golay code, and its cyclic shifts with different orders. We have
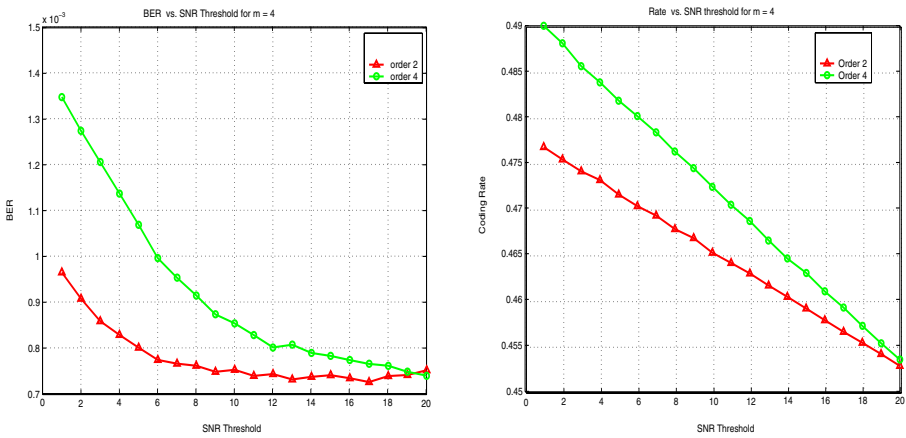

Fig. 3. (a) BER (b) Coding rate vs. SNR threshold or AWGN channels, when $m=4$

demonstrated (Figs. 3.a and 3.b) that by increasing the threshold, both the BER and the coding rate are decreased.

\section{REFERENCES}

[1] R. van Nee, "OFDM Codes for Peak-to-Average Power Reduction and Error Correction," Proc. of IEEE Globecom'96, pp. 740-744, 1996.

[2] X. Li and L. J. Cimini, "Effects of Clipping and Filtering on the Performance of OFDM," Proc. of IEEE Vehicular. Tech. Conf., pp. 1634-1638, May 1997.

[3] M. J. E. Golay, "Complementary Series," IRE Trans. on Information Theory, pp. 82-87, Apr. 1961.

[4] J. A. Davis and J. Jedwab, "Peak-to-Mean Power Control in OFDM, Golay Complementary Sequences, and Reed-Muller Codes.," IEEE Trans. on Information Theory, vol. 45, pp. 2397-2417, Nov. 1999.

[5] S. B. Wicker, Error Control Systems for Digital Communication and Storage. Prentice Hall, New Jersey, 1995.

[6] K. G. Paterson, "Generalized Reed-Muller Codes and Power Control in OFDM Modulation," IEEE Trans. on Comm., vol. 46, pp. 104120, Jan. 2000.

[7] K. G. Paterson and V. Tarokh, "On the Existence and Construction of Good Codes with Low Peak-to-Average Power Ratios," IEEE Trans. on Information Theory, vol. 46, pp. 1974-1987, 2000.

[8] M. Olfat and K. J. R. Liu, "Low Peak to Average Power Ratio Cyclic Golay Sequences in OFDM systems," submitted to IEEE Trans. on Information Theory, June 2003.

[9] M. Sharif, M. G. Alkhansari, and B. H. Khalaj, "On the peak-toaverage power of ofdm signals based on oversampling," IEEE Trans. on Comm., vol. 51, pp. 72-78, Jan. 2003.

[10] M. Olfat and K. J. R. Liu, "Recursive Construction of 16-QAM Super-Golay Codes for OFDM systems," to appear in the Proc. of IEEE Int'l. Conf. on Comm., ICC, 2003.

[11] J. L. Massey, "The Ubiquity of Reed-Muller Codes," Applied Algebra, Algebraic Algorithms and Error-Correcting Codes (Eds. S. Boztas and I. E. Shparlinski), New York, Springer, no. 2227, pp. 1-12, 2001. 